\title{
DESIGN OF A MAGNETIZED IRON MUON SHIELD FOR A HIGH ENERGY NEUTRINO LABORATORY
}

\author{
Y. KANG, K. LEE, A. ROBERTS, S. C. SNOWDON AND D. THERIOT \\ National Accelerator Laboratory, Batavia, Illinois, USA
}

\author{
AND \\ S. L. MEYER \\ Northwestern University, Evanston, Illinois, USA
}

\begin{abstract}
Several new ideas are adduced to the problem of designing muon shielding for a neutrino experimental facility operated at high energy, a problem of interest for the National Accelerator Laboratory (NAL) and CERN-II. We outline a practical calculational procedure which permits the evaluation of the effect of magnetized-iron-lens geometries on the intensity distribution of muons at the detector location. Such relevant effects as range straggling and multiple scattering within and around the lens are included in the evaluation. A specific design is outlined which would increase the maximum energy of bubble chamber beams in the NAL neutrino area from 350 to about $500 \mathrm{GeV}$.
\end{abstract}

\section{FEATURES OF A NEUTRINO FACILITY}

Much of our description here is not new being part of the lore of the trade and having been tacitly assumed in such studies as the Lawrence Radiation Laboratory $200 \mathrm{BeV}$ Accelerator Studies (1964) and the CERN/ECFA Report (1967). We wish,

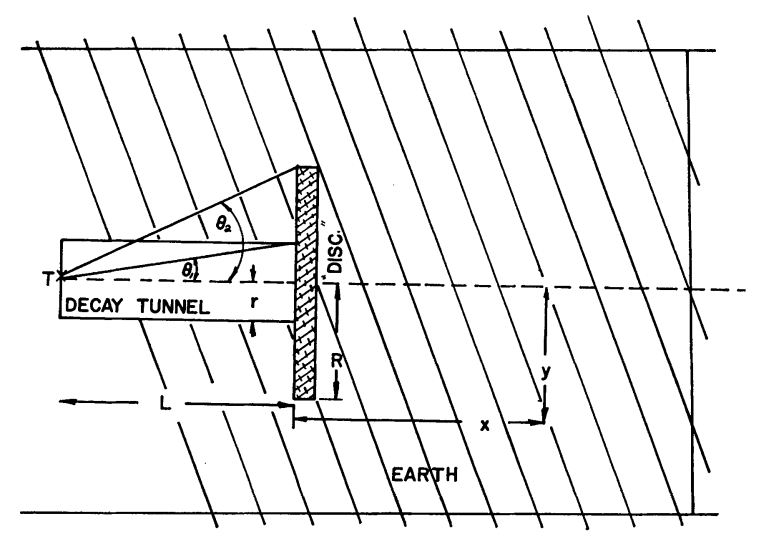

FIG. 1. Simplified diagram of a neutrino beam facility. A proton beam incident from the left produces mesons in the target, $T$; the forward mesons travel along the decay tunnel and many of them decay in flight via leptonic modes that produce neutrinos. The disc is a geometrical construct used for calculating fluxes; it represents a beam dump for both the primary protons and the secondary hadrons. The earth shield serves to range out the muons arising from meson decay in the tunnel. The transverse scale is much exaggerated. however, to provide a basis for non-experts and for our own discussion.

The basic elements of a neutrino facility are shown in Fig. 1. A primary proton beam is impinged on a target $T$ to produce secondary particles, primarily $\pi$ and $K$ mesons. The mesons are allowed to decay in the two-body mode:

$$
\begin{aligned}
\pi^{+(-)} & \rightarrow \mu^{+(-)}+v_{\mu}\left(\bar{v}_{\mu}\right) \\
K^{+(-)} & \rightarrow \mu^{+(-)}+v_{\mu}\left(\bar{v}_{\mu}\right) .
\end{aligned}
$$

The drift (decay) space is usually terminated by a beam stop followed by a massive radiation shield and the detector in series. The neutrinos, of course, being neutral and participating only in weak interactions easily penetrate the beam stop and shield and reach the detector attenuated only by geometry.

If we refer to Fig. 1 we see that the drift space (decay tunnel) has length $L$ and is followed by a beam stop of negligible length, a 'disc' of radius $R$ (for purposes of later discussion), a massive muon shield and a detector area at a distance $x$ downstream of the beam stop. The total space available for the facility is $L+x$. For a given available space we wish to maximize $L$ (up to the point of $\sim 2$ mean lives for the parent mesons) and minimize $x$ in order to maximize the neutrino flux at the detector always subject to the constraint that the charged particle flux at the detector be low enough for personnel safety and bubble chamber operation. 
For monochromatic meson parents of energy $E_{M}$ the neutrino spectrum is flat between essentially zero energy and the energy of the meson, $E_{M}$. The strongly interacting hadrons from the primary interaction are absorbed by the many interaction lengths of material in the beam stop. Likewise, the large number of photons from secondary neutral mesons are soaked up by the many radiation lengths. The shielding problem thus resolves itself into removing the muons associated with meson decays (1) in the drift space.

The differential energy spectrum of muons arising from two-body decays of parent mesons of a given energy $E_{M}$ is constant between muon energy $E_{\mu \text { min }}$ and $E_{\mu \text { max }}$ where

$$
\begin{aligned}
E_{\mu \max } & =\frac{E_{\pi}}{m_{\pi} c^{2}}\left(\frac{m_{\pi}^{2}+m_{\mu}{ }^{2}}{2 m_{\pi}}\right) \mp \frac{P_{\pi}}{m_{\pi}}\left(\frac{m_{\pi}{ }^{2}-m_{\mu}{ }^{2}}{2 m_{\pi}}\right) \\
& \approx{ }_{140 \gamma_{\pi}}^{80 \gamma_{\pi}} \mathrm{MeV} \text { at high energy }
\end{aligned}
$$

for muons arising from pions and

$$
\begin{aligned}
E_{\mu \max } & =\frac{E_{K}}{m_{K} c^{2}}\left(\frac{m_{K}{ }^{2}+m_{\mu}{ }^{2}}{2 m_{\pi}}\right) \mp \frac{P_{K}}{m_{K}}\left(\frac{m_{K}{ }^{2}-m_{\mu}{ }^{2}}{2 m_{\pi}}\right) \\
& \approx 22.6 \gamma_{K} \mathrm{MeV} \text { at high energy } \\
494 \gamma_{K} &
\end{aligned}
$$

for muons arising from kaons.

The muon spectra are shown in Fig. 2a for parent pions of energy $E_{\pi}$ and in Fig. 2 b for parent kaons

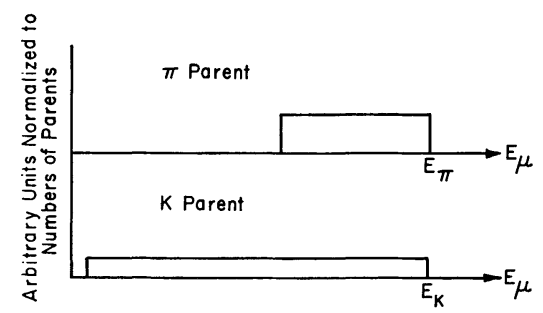

FIG. 2. Muon spectra from two-body decays of pions and kaons. The muon energy spectrum is flat from the highest available energy down to a lower limit given in the text.

of energy $E_{K}$. The muons do not interact strongly and hence can only be ranged out or deflected away from the region to be shielded. The ranges in three possible materials for muons of several energies are shown in Table I.
TABLE I $\dagger$

Muon range in meters-Muon energy in $\mathrm{GeV}$

\begin{tabular}{rccc}
\hline$E_{\mu}$ & $\mathrm{Fe}$ & $\mathrm{Pb}$ & Earth \\
\hline 1 & 0.8 & 0.71 & 2.7 \\
10 & 70.5 & 5.97 & 24.2 \\
50 & 32.0 & 26.6 & 111 \\
100 & 61.9 & 51.2 & 214 \\
200 & 120 & 99.1 & 417 \\
400 & 234 & 193 & 813 \\
500 & 290 & 239 & 1010 \\
\hline
\end{tabular}

† Collision loss only. Cf. D. Theriot, Muon $\mathrm{d} E / \mathrm{d} x$ and Range Tables: Results for Shielding Materials Using Collision Losses Only, NAL TM-260 (1970).

It should be clear that the high energy muons are the background problem. Since it is expected that high energy kaons from the primary interaction will prove to be between 5-20 per cent of the pions and from the fact shown in Fig. 2 that muons from kaon decay have an average energy less than from pion decay, we shall restrict our attention to muons arising from pion decay in our quantitative investigations, ignoring the effects of kaonic muons.

We shall also focus on the problems of operating a bubble chamber in the flux of background muons since the requirements for chamber operation are much more stringent than for personnel safety (of the order of $1 \mu / \mathrm{m}^{2}$-pulse in the bubble chamber whereas personnel safety can tolerate $\sim 10^{5} \mu / \mathrm{m}^{2}$ $\mathrm{sec})$. This requires, for an incident flux of $10^{13}$ interacting protons per second, an attenuation adequate to produce a unit flux $\phi_{0}=10^{-13}$ muons $/ \mathrm{m}^{2}$-interacting proton.

\section{RANGE SHIELDS}

The most conservative position to take is to attempt to provide a shield which can range out muons of the highest possible energy. One has the additional caveat that radiative energy loss, which is subject to large fluctuations, should not be included in the estimation of range lest the straggling be too large. It can be seen readily from Table I that provision of iron shielding quickly becomes very expensive, e.g. to shield $200 \mathrm{GeV}$ requires $120 \mathrm{~m} \times 4 \mathrm{~m} \times 4 \mathrm{~m}=1920 \mathrm{~m}^{3}=15,000$ metric tons or $\$ 3.6 \mathrm{M}$ at $\$ 240 /$ metric ton. If one envisages protecting larger areas, the required tonnage scales accordingly. The estimates are exclusive of excavation and installation costs. 
These shielding considerations have been discussed by Keefe, ${ }^{1}$ Perkins ${ }^{2}$ and other authors. The merits and demerits of using earth shielding were first explored in detail by Camerini and Meyer $^{3}$ who pointed out that earth shielding depressed the low energy part of the neutrino flux spectrum but left the high energy end relatively unchanged from that coming from an iron shield. Considerations of cost and available space led to the construction of the neutrino experimental area at NAL with earth shielding sufficient to range out muons below $\sim 350 \mathrm{GeV}$ energy. The NAL machine, however, is designed to achieve $500-\mathrm{GeV}$ operation without modification. The bubble chamber detector area, moreover, is fixed in position relative to the accelerator and any increase in the maximum energy of muons which are ranged out could not be accomplished with earth shielding. The earth shield case is straightforwardly calculated and results are shown in Fig. 3. On-axis muon fluxes $<10^{-13}$ $\mu / \mathrm{m}^{2}$-interacting proton require an earth shield in excess of $1000 \mathrm{~m}$ length for proton energy of $500 \mathrm{GeV}$.

\section{DEFLECTION SHIELDING-GENERAL CONSIDERATIONS}

The alternative to ranging out muons by a 'brute force' range shield is to deflect the muons away from the personnel area or detector space to be protected. In order to provide economical $\int \mathbf{B} \cdot \mathrm{dl}$ for adequate deflection it is desirable to use magnetized iron for the deflector. It becomes possible to deflect muons sufficiently with much less iron than it would take to absorb them.

Magnetic shields for this purpose have been considered before but the large attenuation factor required, the complexity of the geometry and the difficulties of evaluating multiple scattering effects led most authors ${ }^{2,4}$ to be pessimistic of being able to design anything other than a 'brute force' range shield with very few authors ${ }^{3}$ holding out hope. A major difficulty is the putative need for Monte Carlo calculation of each geometry. This is inconvenient, expensive in computer time, and prone to obscuring the salient design features.

In order to effect an appropriate design of a magnetized iron deflector, various backgrounds due to muons scattering around and through the shield must be considered, since these limit the attenuation possible. Only recently has a simple non-Monte Carlo calculational procedure to estimate these various backgrounds become available. ${ }^{5}$ It is embodied in computer programs such as those developed by Alsmiller et al., Keefe et al., and Nelson. ${ }^{6}$ We have used the Alsmiller program in our work. This muon transport program calculates the intensities of muons in a semi-infinite homogeneous medium, using the theory of Eyges ${ }^{7}$ to include the effects of both multiple Coulomb scattering and collision energy loss.

The basis of our design technique is thus to identify and compute the contribution of each background muon source, and then to reduce the contribution of each to the necessary level. We use only analytic techniques (with one exception, wherein the Monte Carlo technique was invoked to verify that a specific contribution was negligible). Thus we have been able easily to vary the relevant parameters, and to obtain the total resultant muon flux at the detector.

The Alsmiller program requires as input a given spectrum of incident muons. We used the Trilling formula with current best parameters ${ }^{8}$ for parent hadron production, and from this derived the decay muon spectrum, neglecting the kaon contribution because the muons are in general less energetic. Since the results will be quoted 'per interacting proton', the lighter targets will produce somewhat higher energy secondaries than will heavier targets. We have concentrated on production from beryllium so as to be conservative. To calculate muon ranges, we rely on the observation of $\operatorname{Roe}^{9}$ that a conservative combination of collision-energy-loss and direct-pair-production-energy-loss chosen so as to be safe against fluctuations of 7 standard deviations in the Gaussian sense obviates straggling corrections, and is equivalent to use of the total collision energy loss alone if one starts with $500 \mathrm{GeV}$ muons. $^{9}$ We have, therefore, used just the total collision loss without radiation loss and without subsequent straggling corrections. Our results are conservative in this regard down to attenuations of $10^{12}-10^{13}$. Since only the most energetic muons produced can penetrate the shield, it is frequently. possible to ignore the lower half of the production spectrum and thus to simplify calculations. The Trilling formula we have used tends to overestimate 
the high energy portion so that our results are conservative.

We have used the NAL values for the shielding geometry: a decay tunnel $400 \mathrm{~m}$ long, $0.45 \mathrm{~m}$ in radius, followed by a beam dump, a circular 'disc', and earth shielding (see Fig. 1). The 'disc' represents either an iron plug or a magnetic deflector. We assume $10^{13}$ interacting protons/pulse in the neutrino target, which is the desired level of operation for wide-band neutrino experiments, and express our results in terms of residual muons $/ \mathrm{m}^{2}$ interacting proton.

\section{DEFLECTION SHIELDING- CALCULATIONS}

The Alsmiller program gives the muon flux as a function of distance off-axis for a semi-infinite medium, with a known source function. The effect of magnetic deflection is obtained by calculating the angle of deflection, $\theta_{d}$, and taking a new direction, which makes an angle $\theta_{d}$ with the undeflected beam, as the axis for the Alsmiller distribution. The previous axis, the undeflected direction, then will have intensities that can be read off the Alsmiller curves by superposing on them a line showing the new deflected axis, and reading off the reduced intensities at the corresponding off-axis distances. This is shown in Fig. 5. In practice, the required magnetic deflection is obtained by using the Alsmiller distribution and determining what angle is required to bring the intensity at the given depth in the shield down to the required value.

In principle this procedure ought to be applied to several different energy bins independently, since the magnetic deflection depends on the momentum. We have deliberately ignored this and made the calculations on the assumption that all muons, however soft, undergo the same magnetic deflection; this estimate is perhaps not as conservative as it sounds, since the residual muons at the detector all arise from the upper end of the muon spectrum.

\section{Sources of Muons}

We enumerate three sources which contribute to the net muon flux at the detector and note that this analysis is applicable to two types of hybrid shields: magnetized-iron-deflection-plus-earth shields as well as earth-plus-iron-plug. The geometry of interest is shown in Fig. 1. We consider that the 'disc' at the end of the decay tunnel represents either a magnetic deflector or an iron plug. Three different sources of muons that contribute to the net flux at the detector are as follows:

(1) Muons are emitted within an angular range $\theta<\theta_{1}$ where $\tan \theta_{1}=r / L$ and strike the disc at a radius less than or equal to $r$. These muons pass through no material before the disc and hence are all transported through the disc. They may thereafter scatter but we refer to this muon contribution as TRANSMISSION (I) only. These muons are characterized by large energies since they are produced at forward angles and we must reduce the flux of these muons by deflecting them away from the detector or by ranging them out with a combination of earth and iron.

(2) Muons produced with angles $\theta_{1} \leqq \theta \leqq \theta_{2}$ where $\tan \theta_{2}=R / L$ would, if propagated along straight lines, strike the disc at radii greater than $r$ and less than or equal to $R$. These muons, however, must pass through a length of earth shielding medium which varies between zero and (approximately) $L(1-r / R)$. Muons in this region can make two kinds of contribution to the net muon flux at the detector since they can scatter and pass around the disc (and scatter back to the detector) or pass through the disc. The muons which pass through the disc make a contribution to the net muon flux similar to that of TRANSMISSION (I) and we call this contribution TRANSMISSION (II). The muons which scatter around the disc may make a contribution GROUNDSHINE (II).

(3) Muons produced at angles greater than $\theta_{2}$ will in general produce only a contribution to the scattered flux by passing around the disc. This contribution is GROUNDSHINE (III). Muons from this third production region can also scatter and pass through the disc making a contribution TRANSMISSION (III).

There is little point to reducing one contribution if another is larger. In all cases, there is no reason to reduce the muon flux to a level below that produced by neutrino interactions in the shield themselves producing muons. We estimate this level to be 2-4 $\phi_{0}$ depending on the distance between the end of the shield and the detector. 
Thus, there is a natural point of diminishing returns.

\section{Summary of Procedure}

From a simple viewpoint, the transmission muons are treated with either magnetic deflection or ranging in iron while the groundshine muons are ranged out in earth. Qualitatively, as the radius of the disc is increased, the groundshine muons become less in intensity and, more important, softer in energy. The groundshine muons are thus ranged out in smaller earth shields for larger disc radii. The radius of the disc is thus an important parameter to vary along with its distance from the target.

The contribution of TRANSMISSION (I) is straightforwardly calculated. The contribution of TRANSMISSION (II) has been conservatively overestimated by assuming that all muons produced in the (II) angular region are transmitted through the disc. The Alsmiller program suffices to calculate TRANSMISSION (I) and TRANSMISSION (II). The contribution of TRANSMISSION (III) is neglected since the muons from region III are in general of lower energy than those in the other regions and we shall thus assume that these muons are readily removed by magnetic deflection and/or direct ranging in iron. The contribution of GROUNDSHINE (III) is likewise readily calculable by the Alsmiller program.

The program does not, however, easily lend itself to a calculation of GROUNDSHINE (II) since the geometry for this is not homogeneous. However, we have calculated with the Alsmiller program the number of muons with production angles $\theta \leqq \theta_{2}$ which scatter sufficiently to miss the disc. We consider in the category only muons with energy $\geqq E_{\max }$ where $E_{\max }$ is sufficient to penetrate the residual shield based on collision energy loss alone over the shortest possible path. This permits the evaluation of an upper limit to that of GROUNDSHINE (II) which could make a contribution to the background at the detector. These may scatter back but will be spread over an area such that the flux at the detector will be less by a factor depending on geometry. This yields an upper limit to GROUNDSHINE (II). The contribution GROUNDSHINE (II) may also be calculated by Monte Carlo methods.

\section{RESULTS FOR SOME SIMPLE GEOMETRIES}

Let us consider the flux of interest in our discussion to be

$$
\phi_{0}=10^{-13} \text { muons } / \mathrm{m}^{2} \text {-interacting proton }
$$

with the Trilling formula applied to a beryilium target.

Figure $3 \mathrm{a}$ shows that the earth shield required to reduce the on-axis muon flux to $\phi_{0}$ is in excess of $1100 \mathrm{~m}$ length for a $600 \mathrm{~m}$ decay length. Likewise Fig. $3 \mathrm{~b}$ shows that more than $1000 \mathrm{~m}$ of earth are

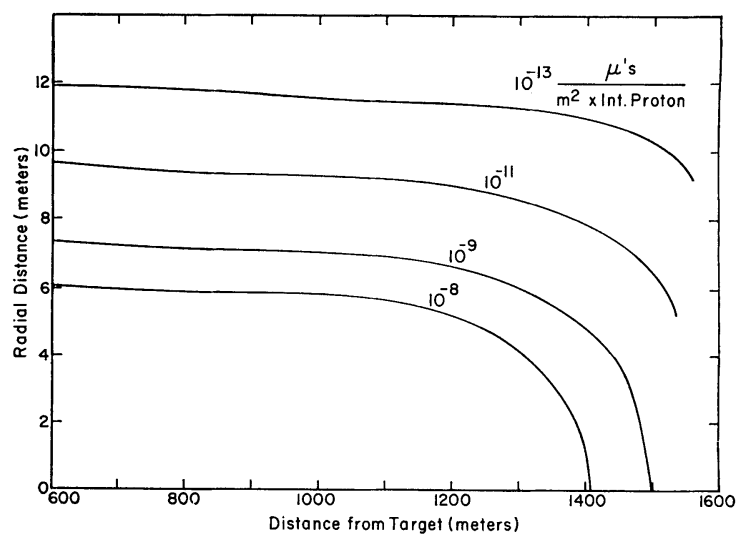

FIG. 3a. Isoflux contours for muons produced from pion decay in a $600 \mathrm{~m}$ long decay tunnel, inside an infinite earth shield, as a function of distance downstream and of radial distance from the beam line. The sharp cutoff at the end is due to the ranging out of the muons in the earth. Calculations here and in subsequent maps are based on the Trilling formula for meson yield from beryllium and a primary proton energy of $500 \mathrm{GeV}$.

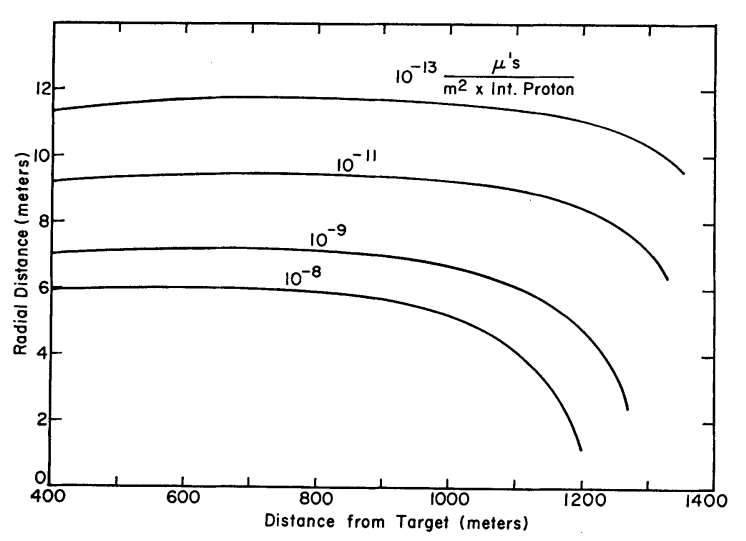

FIG. 3b. Same as Fig. 3a, but for a decay tunnel $400 \mathrm{~m}$ long. 
required for a decay length of $400 \mathrm{~m}$. These results have been verified with use of the Alsmiller program and, within a factor of 2 in $\phi_{0}$, by direct Monte Carlo calculation.

Figure 4a shows the Alsmiller program's results for the calculation of GROUNDSHINE (III) in the case of a disc $1.5 \mathrm{~m}$ in radius placed at the end of a $600 \mathrm{~m}$ decay tunnel. For this case, the on-axis flux contribution from this source is reduced to $\phi_{0}$ after less than $550 \mathrm{~m}$ of earth.

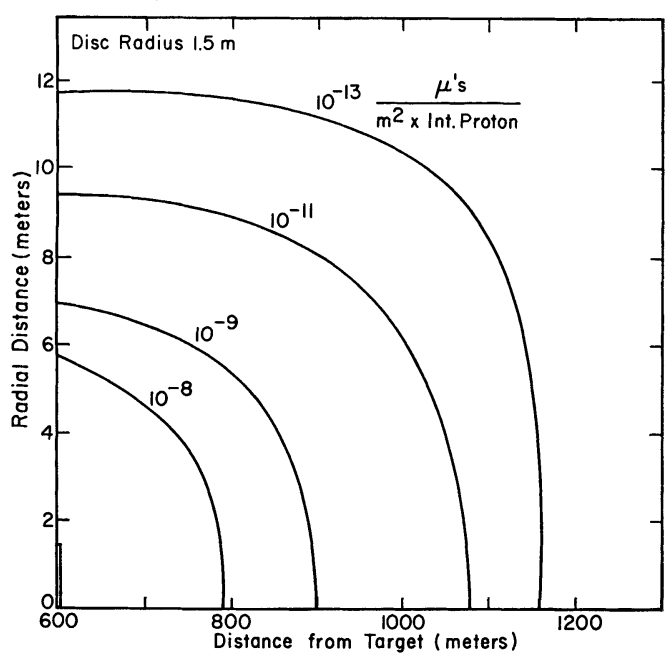

FIG. 4a. Muon isoflux contours for GROUNDSHINE (III), muons scattered around the disc. The decay tunnel is $600 \mathrm{~m}$ long, the disc radius is $1.5 \mathrm{~m}$.

As earlier discussed, GROUNDSHINE (II) cannot be explicitly calculated using the Alsmiller program. For muon energies in the decay tunnel in excess of $350 \mathrm{GeV}$, however, we estimate that $10^{-11}$ muons per interacting proton emerge from around the disc. These will spread over an area such that the flux per unit area at the detector is $\sim 50$ times less. This estimate yields $2 \phi_{0}$ at the detector as an upper limit to GROUNDSHINE (II) in this case. The contribution GROUNDSHINE (II) makes no contribution down to the level of $\phi_{0}$ for any earth shield length in excess of $550 \mathrm{~m}$ when the disc is placed at the end of the decay tunnel. Monte Carlo calculation verifies this.

Figure 5a shows isoflux curves for TRANSMISSION (I) plus an overestimate of TRANSMISSION (II) for the case of $R=1.5 \mathrm{~m}$ and
$L=600 \mathrm{~m}$. The muons in this case all pass through the disc. If we consider that the disc is a magnetic deflector which can produce a deflection of $22 \mathrm{mrad}$ on the highest energy muons, we can get an overestimate of the on-axis flux contribution from this

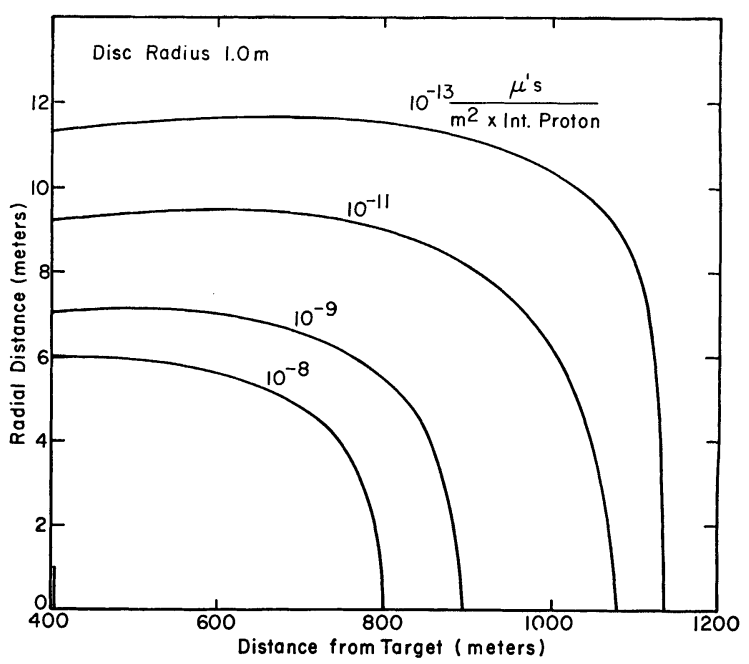

FIG. 4b. Same as Fig. 4a, except tunnel is $400 \mathrm{~m}$ long, disc radius $1.0 \mathrm{~m}$.

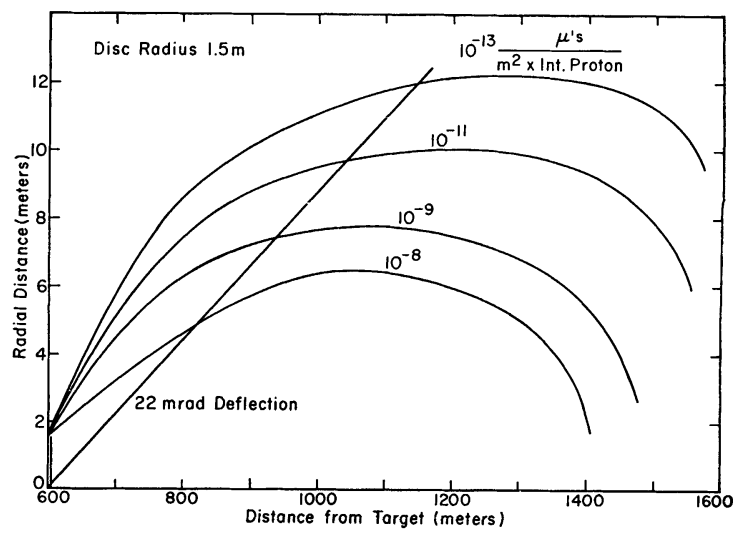

FIG. 5a. Method of calculating TRANSMISSION with a deflecting lens at the position of the disc. The curves are the isoflux contours for muons that penetrate the $1.5 \mathrm{~m}$ radius disc. The straight line labelled 22 mrad deflection, shows the axial intensity distribution of this component of muons, on the assumption that the lens has bent all the muons through this angle, so that the axis of the muon distribution now makes a 22 mrad angle with the former beam axis. The on-axis intensity at $1200 \mathrm{~m}$ is $10^{-7}$ with the lens off; with the lens on, it is less than $10^{-13}$. The TRANSMISSION contribution to on-axis intensity is thus negligible with the lens on. 
source as indicated on the figure. The on-axis flux is reduced to less than $\phi_{0}$ after $550 \mathrm{~m}$ of earth.

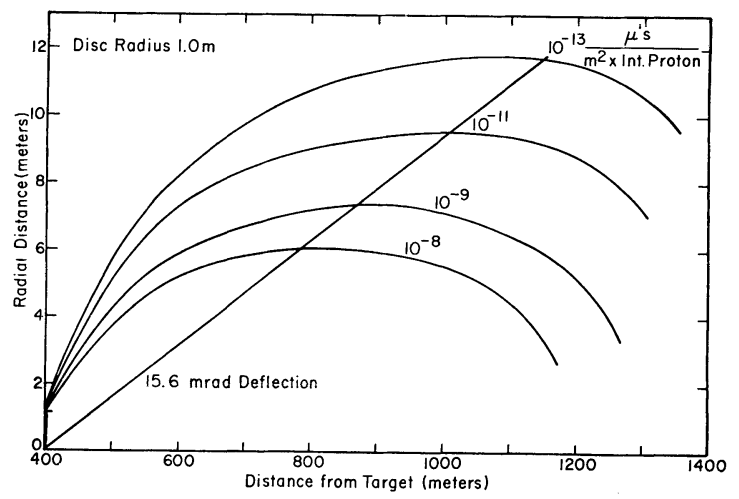

FIG. 5b. Same as Fig. 5a, but for $400 \mathrm{~m}$ decay tunnel and a disc radius of $1.0 \mathrm{~m}$. Now the required angular deflection of the muon beam axis is only $15.6 \mathrm{mrad}$.

Figures $4 \mathrm{~b}$ and $5 \mathrm{~b}$ show the results for the case $R=1.0 \mathrm{~m}$ and $L=400 \mathrm{~m}$. Table II summarizes the results for these simple geometries.

TABLE II

Results for some simple geometries

\begin{tabular}{cccc}
\hline $\begin{array}{c}\text { Decay } \\
\text { tunnel } \\
\text { length }\end{array}$ & $\begin{array}{c}\text { Disc } \\
\text { radius }\end{array}$ & $\begin{array}{c}\text { Maximum deflection } \\
\text { angle needed for } \\
\text { highest energy } \\
\text { muons }\end{array}$ & $\begin{array}{c}\text { Length of earth } \\
\text { shield needed to } \\
\text { reduce on-axis } \\
\text { flux to } \phi \leqq \phi_{0}\end{array}$ \\
\hline $600 \mathrm{~m}$ & $1.5 \mathrm{~m}$ & $22 \mathrm{mr}$ & $550 \mathrm{~m}$ \\
$400 \mathrm{~m}$ & $1.0 \mathrm{~m}$ & $16 \mathrm{mr}$ & $750 \mathrm{~m}$ \\
\hline
\end{tabular}

\section{PRACTICAL REALIZATION OF A MAGNETIC DEFLECTOR}

While it would be desirable to use a transversely magnetized block of iron as a deflector, the magnetization that can be obtained in a short sample is too low to be useful with any reasonable excitation current. We thus must consider toroidal magnetization where the iron is surrounded by a current-carrying conductor to obtain flux lines whose path is entirely in iron and which require correspondingly low magnetization currents.

To be specific we may consider a design which would permit the construction of a 'disc'. The deflector would now consist of two parts: the magnetized iron deflector or lens, and an axial iron 'plug' to protect the open center of the lens.
The deflector is a stack of soft iron. It is magnetized by an axial current passing through a gap, the return legs of the winding being outside the iron. The toroidal magnetization produced has the flux lines approximately circular and coaxial with the beam. Particles traveling more or less parallel to the axis are, therefore, deflected either away from the axis or towards it, depending on their sign of charge. The deflector is thus a lens, converging for one sign of particle and diverging for the other as indicated schematically in Figs. 6a, 6b. In either

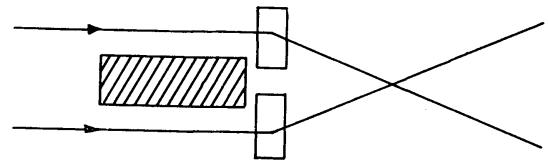

(a)

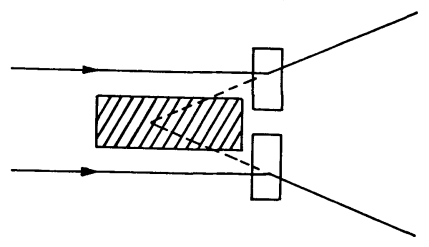

FIG. 6. Schematic optical properties of toroidal magnetic lens. In (a) the lens is converging and particles diverge after passing through a real focus. For the opposite sign of particles, (b) shows the lens to be diverging with a virtual focus.

case, the particles eventually diverge from the real (Fig. 6a) or the virtual focus (Fig. 6b). The highest energy muons present are deflected sufficiently to miss the axially located detector downstream.

The deflector thus focuses the muons into a diverging cone. It should be recognized that the lateral elements of the cone may give increased radiation intensities at ground level. However, the radiation is local where it leaves the earth shield, rapidly diverging and not very intense.

The magnetic deflection $\theta_{d}$ to be supplied by the lens must be sufficient to produce the desired decrease of length in the axial shielding required to reduce the transmitted flux to $\phi_{0}$. However, the iron of the lens will itself introduce scattering and, since the mean scattering angle varies as the square root of the lens thickness while the magnetic deflection is linear in it, the ratio of magnetic to scattering deflection increases only as the square root of the lens thickness and is independent of momentum. (This result is well-known to designers of cosmic-ray muon spectrometers.) For the $15-\mathrm{m}$ length we require to obtain sufficient 
deflection, the mean scattering angle is about 6 per cent of the deflection angle. The effect of scattering within the lens can be estimated by varying the assumed deflection angle in Fig. 5 and noting the effect on axial intensity; if the effect of decreasing the deflection angle does not increase more rapidly than the scattered intensity falls off with angle, the process is a converging one and a satisfactory deflection angle can be found. This is fortunately the case, and the effects of scattering as well as misalignments, etc. are included by providing for a magnetic deflection large enough to include several times the mean scattering angle.

The 'plug' is a stack of unmagnetized iron large enough in cross section to protect the center of the lens and placed on the beam axis upstream of the deflector. The design criteria for the system plug + lens are as follows:

1. Particles which traverse the entire plug emerge with too little energy to penetrate to the detector.

2. Particles which miss the plug pass through the magnetic lens and are deflected sufficiently to give the requisite attenuation in muon flux at an axially located detector at the end of the shield.

3. Particles which enter the plug but are scattered out of it should either: $a$. strike the magnetic lens and be adequately deflected or, $b$. if they miss the deflector, have too low an energy to reach the detector.

4. Particles which miss both the plug and the magnetic lens should have too low an energy to reach the detector.

The plug has two major effects. It slows down the muons which traverse it and, for all muons not scattered out of it, reduces their energy to the point that they do not reach the bubble chamber even if they traverse the deflector on axis (and, hence, are not deflected). On the other hand, most muons are scattered out of it, and are then subject to defocussing by the magnetic lens. The scattered muons must then only be sufficiently deflected by the magnetized iron to compensate for the additional outward deflection due to scattering from the plug. (This applies only to the converging case; for the diverging lens the additional scattering is helpful and increases the divergence of the beam.)
The mean scattering angle produced by the iron plug is proportional to the square root of the length traversed, while the loss of momentum, and hence the increased deflection in the lens, are linear in that quantity. There is, therefore, a minimum length above which the increased deflection after the lens caused by the plug will always exceed the scattering caused by the plug. For iron plugs, this length is $20-30 \mathrm{~m}$ over the entire momentum region of interest. For lengths less than the minimum the escape probability of the scattered muon is low, and the scattering angle small, so that the net deflection deficit produced by the plug rarely exceeds $1 \mathrm{mrad}$. Thus design criterion $3 a$ above is easily satisfied. Muons which are scattered out of the plug at so large an angle that they miss the deflecting lens are of too low an energy to reach the detector area, thereby satisfying criterion $3 b$.

\section{A SPECIFIC DESIGN OF MAGNETIC DEFLECTOR}

For purposes of illustration we discuss a specific design which we have investigated at the National Accelerator Laboratory. The toroidal magnet is to be built from 1200 tons of iron obtained from the Rochester Cyclotron together with another 350 tons of flat plate to provide a magnetic lens $3 \mathrm{~m} \times 3 \mathrm{~m} \times$ $16 \mathrm{~m}$ length as shown in Figs. 7 and 8 . Figure 9a

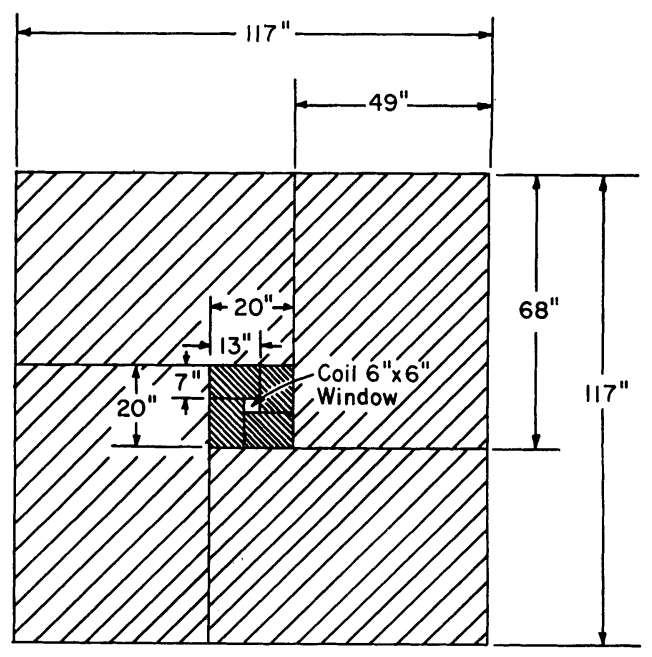

FIG. 7. Cross section of the proposed lens design, using the (68 in. $\times 49$ in.) forgings available from the Rochester synchro-cyclotron. 


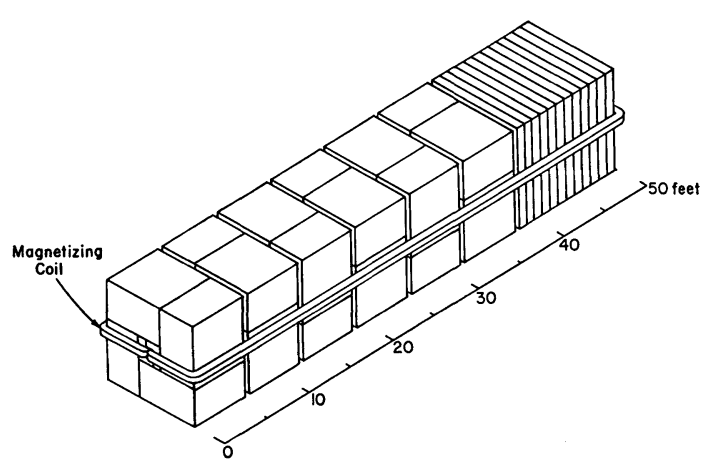

FIG. 8. Perspective view of the proposed lens, which will be $50 \mathrm{ft}$ long, $10 \mathrm{ft}$ by $10 \mathrm{ft}$ in cross section. The magnetization is produced by axial currents, with the return legs wound on the outside faces.

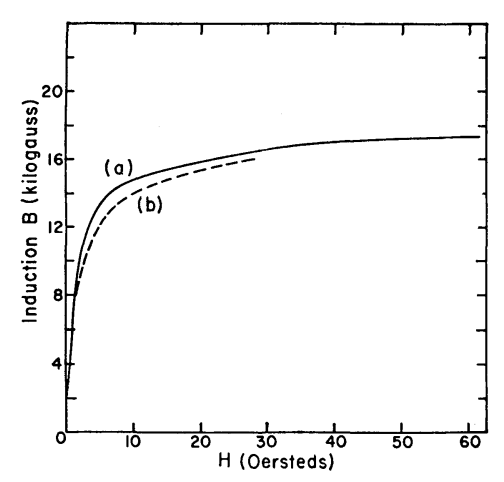

FIG. 9. (a) is the $B-H$ curve for iron from the Rochester synchro-cyclotron. (b) is the $B-H$ curve for U.S. Steel low-carbon scrap steel, as presently available at $\$ 65 /$ ton. shows the magnetization curve for the Rochester Cyclotron iron while Fig. 9b shows that for iron which is commercially available (U. S. Steel, Gary, Indiana) in large quantities at $\sim \$ 65 /$ ton. The effective permeabilities of both kinds of iron are comparable and adequate for the purpose. The magnet is energized by an axial current passing through a gap 6 in. $\times 6$ in. Upstream of the magnet is an iron plug $16 \mathrm{in}$. square and $100 \mathrm{~m}$ long. With a coil containing $4 \frac{1}{2}$ tons of copper, $130 \mathrm{~kW}$ of power will provide $17 \mathrm{kG}$ in the iron assuming stacking which leave quarter-inch gaps between iron blocks (in the direction of the flux lines). The required power scales directly with the average gap spacing.

The magnet itself is buried in an earth berm $320 \mathrm{~m}$ past the end of the decay pipe, $720 \mathrm{~m}$ from the target. The iron plug begins $220 \mathrm{~m}$ past the end of the decay pipe. These elements could not be located closer to the end of the decay pipe because of various magnet enclosures and beam pipes which are buried in the berm after the decay pipe.

The construction geometry of the neutrino area as drawn in Fig. 10 with this magnet in place was tested by means of a Monte Carlo program. The bubble chamber that we are interested in protecting is located approximately $1300 \mathrm{~m}$ downstream from the target and $900 \mathrm{~m}$ after the end of the decay pipe. The $900 \mathrm{~m}$ is occupied by shielding berm, magnet enclosures, beam pipes, and cuts for other experimental areas, having a total effective shielding

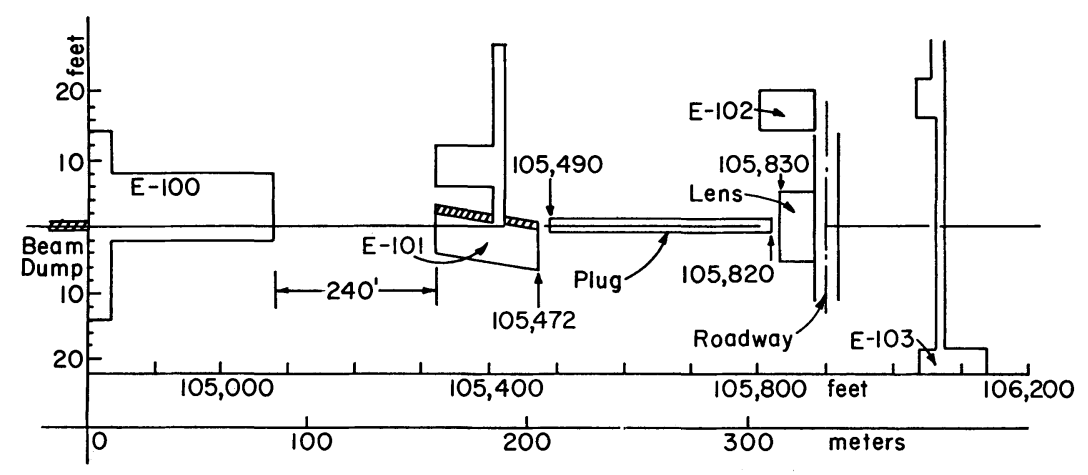

FIG. 10. A simplified map of the NAL neutrino area, showing the location of the various components of the muon deflecting shield. Note the differing transverse and longitudinal scales. The left-hand end of the drawing starts at the beam dump, which is the end of the 400-m decay tunnel. The scale in feet shows surveyors' readings; the dimensions in meters are also shown. The iron plug, 16 in. square in cross section and $330 \mathrm{ft}$ long, starts downstream of building E-101 and is followed by the lens, located near building E-102 for convenience in servicing. 
length of $716 \mathrm{~m}$ of soil when the various voids are taken into account. This corresponds to a range shield for muons of $350 \mathrm{GeV}$.

When the magnet was turned off, the muon flux at the bubble chamber was evaluated to be $0.43 \times 10^{-8}$ muons $/ \mathrm{m}^{2}$-interacting proton. When the magnet was activated to a field strength of 16 $\mathrm{kG}$, these muon fluxes dropped to the level of $1.3 \times 10^{-11}$ muons $/ \mathrm{m}^{2}$-interacting proton for 500 $\mathrm{GeV}$ protons, an improvement of approximately 330, although still higher than desirable for operation of a bubble chamber. All of the muons that reached the vicinity of the bubble chamber were found to be of the GROUNDSHINE (II) or GROUNDSHINE (III) type, predominantly GROUNDSHINE (III). All muons of the TRANSMISSION type were successfully deflected away from the region of the bubble chamber. This was to be expected because our simple considerations calculated the deflections necessary for the highest energy muons and hence would give an overestimate of the total flux which is mainly lower energy muons. A few further studies were carried out varying the parameters of the size of the magnet and the incident proton energy. If the magnet were increased in its transverse dimension to $3.65 \mathrm{~m} \times$ $3.65 \mathrm{~m}$, the flux summed over the region of the bubble chamber would correspond to $2.3 \times 10^{-12}$ muons $/ \mathrm{m}^{2}$-interacting proton; for $4.26 \mathrm{~m} \times 4.26 \mathrm{~m}$, the flux would be $3.7 \times 10^{-13}$. If, instead, the magnet was left alone and the incident proton energy varied, the flux for $475 \mathrm{GeV}$ protons incident would be $1.4 \times 10^{-12}$; for $450 \mathrm{GeV}$, the flux would be $1.6 \times 10^{-13}$. All of the calculations were carried out using the Trilling formula for a Be target. Compared to other production models such as $\mathrm{CKP}^{10}$ or Hagedorn-Ranft ${ }^{11}$ the Trilling formula generally overestimates the production of high energy pions, ${ }^{12}$ therefore these estimates should be regarded as pessimistic.

\section{CONCLUSION}

In summary we feel that our proposed design represents a reasonable compromise with high probability of providing the desired muon flux levels for operation of a bubble chamber with 450-500 GeV protons on target, thus increasing the useful maximum energy approximately 100-150
$\mathrm{GeV}$ above that available from that passive range shield alone. Any stronger statement will have to wait for the actual operation of such a facility.

\section{REFERENCES}

1. D. Keefe, Neutrino Beams at High Energies, Lawrence Radiation Laboratory Report, $200 \mathrm{BeV}$ Accelerator: Studies on Experimental Use, Vol. 1, p. 311 (1964).

2. D. H. Perkins, CERN/ECFA Report, 67/16, Vol. II, 1967.

3. U. Camerini and S. L. Meyer, National Accelerator Laboratory 1968 Summer Study, Vol. 1, p. 157.

4. D. D. Jovanovic, The Neutrino-Muon Facility at the $200 \mathrm{BeV}$ Accelerator, Lawrence Radiation Laboratory Report UCRL-16830, Vol. 3, p. 83 (1966).

5. D. Theriot and M. Awschalom, Muon Shielding: Studies of Homogeneous Shielding for a Neutrino Facility, NAL TM-259 (1970).

Y. Kang, A. Roberts and S. L. Meyer, Design of Muon Shields Using Magnetized Iron Deflection, NAL TM-263 (1970).

Y. Kang, D. Theriot and S. L. Meyer, Design of Muon Shields: Some General Considerations with Special Reference to Magnetized Iron Deflection, NAL TM-267 (1970).

Y. W. Kang, A. Roberts, D. Theriot and S. L. Meyer, IEEE Trans. Nucl. Sci., NS-18, No. 3, 753 (1971).

Y. W. Kang, A. Roberts, S. C. Snowdon, D. Theriot and S. L. Meyer, A Proposal for Achieving $500 \mathrm{GeV}$ Operation with the 15-Ft. Bubble Chamber, NAL TM-300 (1971).

6. R. G. Alsmiller, M. Leimdorfer and J. Barish, Oak Ridge National Laboratory Report ORNL-4322 (1968); D. Keefe and C. M. Noble, UCRL 18117 (1968); W. R. Nelson, Nucl. Instr. and Meth., 66, 293 (1968).

7. L. Eyges, Phys. Rev., 74, 1534 (1948).

$$
\text { 8. } \begin{aligned}
\frac{\mathrm{d}^{2} N}{\mathrm{~d} p \mathrm{~d} \Omega}= & A_{1} p^{2} \exp \left\{\frac{-A_{2} p}{\sqrt{p_{0}}}-A_{3} p \sqrt{p_{0}} \theta^{2}\right\} \\
& +A_{4} \frac{p^{2}}{p_{0}} \exp \left\{-A_{5}\left(\frac{p}{p_{0}}\right)^{2}-A_{6} p \theta\right\}
\end{aligned}
$$

where $p_{0}=$ primary beam momentum and the parameters of the Trilling formula for $\pi \pm$ production from $\mathrm{Be}, \mathrm{Al}, \mathrm{Cu}$ and $\mathrm{Pb}$ targets are as follows:

\begin{tabular}{cccccccc}
\hline Particle & Target & $A_{1}$ & $A_{2}$ & $A_{3}$ & $A_{4}$ & $A_{5}$ & $A_{6}$ \\
\hline$\pi^{+}$ & $\mathrm{Be}$ & 3.52 & 4.15 & 4.56 & 3.49 & 9.87 & 4.04 \\
$\pi^{-}$ & $\mathrm{Be}$ & 3.52 & 4.15 & 4.56 & 1.01 & 9.87 & 4.04 \\
$\pi^{+}$ & $\mathrm{Al}$ & 3.88 & 4.15 & 4.56 & 3.04 & 10.0 & 3.91 \\
$\pi^{-}$ & $\mathrm{Al}$ & 3.88 & 4.15 & 4.56 & 0.82 & 10.0 & 3.91 \\
$\pi^{+}$ & $\mathrm{Cu}$ & 4.13 & 4.15 & 4.56 & 2.47 & 9.68 & 4.01 \\
$\pi^{-}$ & $\mathrm{Cu}$ & 4.13 & 4.15 & 4.56 & 0.67 & 9.68 & 4.01 \\
$\pi^{+}$ & $\mathrm{Pb}$ & 3.43 & 4.15 & 4.56 & 1.88 & 9.94 & 3.85 \\
$\pi^{-}$ & $\mathrm{Pb}$ & 3.43 & 4.15 & 4.56 & 0.56 & 9.94 & 3.85 \\
\hline
\end{tabular}

J. Ranft and T. Borak, Improved Nucleon-Meson Cascade Calculations, NAL FN-193 (1969). 
9. B. Roe, Nucl. Instr and Methods, 92, 257 (1971). At $500 \mathrm{GeV}$, seven standard deviation 'safety' requires the use of $\left.0.80 \frac{\mathrm{d} E}{\mathrm{~d} x}\right|_{\text {collision }}+\left.0.52 \frac{\mathrm{d} E}{\mathrm{~d} x}\right|_{\text {pair }}$ where the collision energy loss for $0-500 \mathrm{GeV}$ is $\left.\frac{\mathrm{d} E}{\mathrm{~d} x}\right|_{\text {collision }}=$ 2.26 and energy loss due to direct pair production is $\left.\frac{\mathrm{d} E}{\mathrm{~d} x}\right|_{\text {pair }}=0.98$. The weighted sum is $2.30 \mathrm{MeV} / \mathrm{g}$ $\mathrm{cm}^{2}$ for $\frac{\mathrm{d} E}{\mathrm{~d} x} \mid$ which is $\left.\frac{\mathrm{d} E}{\mathrm{~d} x}\right|_{\text {collision }}$.
10. G. Cocconi, L. J. Koester and D. H. Perkins, UCRL10022, 1961 (p. 107).

11. R. Hagedorn and J. Ranft, CERN/ECFA 67/16, Vol. 1, 1967, p. 70

12. T. G. Walker, NAL 1968 Summer Study, Vol. 2, p. 59.

Received 1 May 1972

and in final form 10 July 1972 